\title{
Performance of hydrothermal PZT film on high intensity operation
}

\author{
Takefumi Kanda a,*, Minoru Kuribayashi Kurosawa ${ }^{\text {, }}$, \\ Hidehiko Yasui $^{\mathrm{a}}$, Toshiro Higuchi ${ }^{\mathrm{a}}$ \\ ${ }^{a}$ Department of Precision Machinery Engineering, Graduate School of Engineering, \\ The University of Tokyo, 7-3-1 Hongo, Bunkyo-ku, Tokyo 113-8656, Japan \\ ${ }^{\mathrm{b}}$ Department of Advanced Applied Electronics, Interdisciplinary Graduate School of Science and Engineering, \\ Tokyo Institute of Technology, 4259 Nagatsuta-cho, Midori-ku, Yokohama 226-8502, Japan
}

\begin{abstract}
The deposition processes of PZT thin films have been described in many reports, yet there have been no descriptions of the performance of PZT films at high intensity operation. Hydrothermally-deposited PZT film transducers were examined at large vibration amplitude levels at resonance frequencies. It was demonstrated that the hydrothermal PZT film expresses superior performance with regard to linearity and maximum vibration velocity. The linearity of frequency responses at around the resonance frequency was excellent and superior to bulk material up to high intensity operation. The characteristics of longitudinal transducers using films formed under two deposition processes

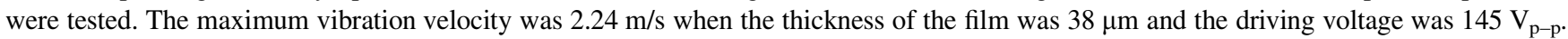
This velocity value was much larger than bulk PZT materials for high power applications. (C) 2001 Elsevier Science B.V. All rights reserved.
\end{abstract}

Keywords: PZT; Thin film; Hydrothermal method; Vibration velocity; Polycrystal structure

\section{Introduction}

Several kinds of deposition processes for piezoelectric materials have been developed for many applications in electronic devices and electro-mechanical devices. Especially, in actuators and sensors, high electro-mechanical coupling materials are preferred. Hence, the piezoelectric factors such as $d_{31}, d_{33}$, and/or $e_{31}$ were measured to evaluate the material's performance. Correctly, these factors are measured in the evaluation of the material's performance, including a device's static characteristics. For example, these factors are used to calculate the sensitivity of a sensor or electro-mechanical transformation ratio.

However, we cannot determine the dynamics of devices based on these statically defined piezoelectric factors. For example, in the case of ultrasonic motors, the maximum rotation speed is limited by the vibration velocity of the transducer. Therefore, in order to determine the limit of the vibration velocity, it is important to know the device's mechanical output. The static factors indicate only the ratio between an electric port and a mechanical port, providing little information regarding the device's performance under working conditions.

\footnotetext{
${ }^{*}$ Corresponding author. Fax: +81-45-924-5593.

E-mail address: kanda@intellect.pe.u-tokyo.ac.jp (T. Kanda).
}

Recently, in the world of normal size piezoelectric devices for power application such as piezoelectric transformers, ultrasonic processing tools, and ultrasonic motors, the dynamic performance of piezoelectric materials have attracted attention. Linearity and dynamic range are also important factors for high performance electro-mechanical devices.

Hydrothermally-deposited PZT film transducers were examined at large vibration amplitude levels at around resonance frequencies. It was demonstrated that hydrothermal PZT film expresses superior performance with regard to linearity and maximum vibration velocity. The linearity of frequency responses at around the resonance point was excellent and superior to bulk material up to high intensity operation. The maximum vibration velocity of a longitudinal transducer was $2.24 \mathrm{~m} / \mathrm{s}$.

\section{Hydrothermal deposition of PZT film}

We have reported a micro-ultrasonic motor [1], a vibration touch probe sensor [2,3], and an ear horn [4], all of which use hydrothermal PZT film. The hydrothermal method utilizes the chemical reaction between titanium base metal and the melted ions at high temperatures in aqueous solutions. The chemical reaction is carried out in the solution 


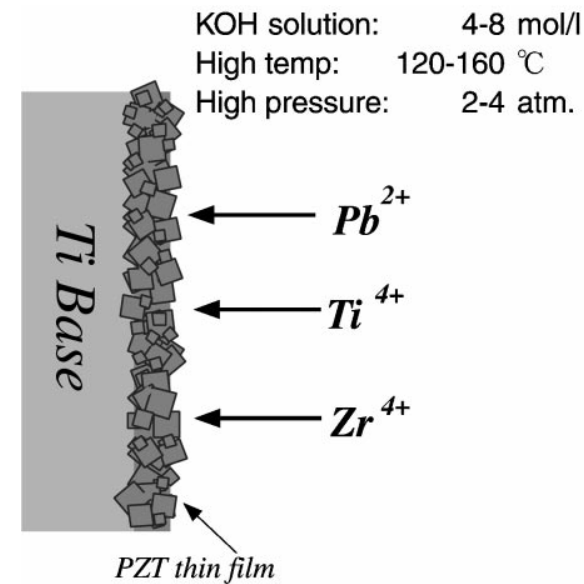

Fig. 1. Schematic of the hydrothermal method for PZT thin film.

as shown in Fig. 1. Other beneficial characteristics of the hydrothermal method include its ability to deposit thick PZT film, automatic polarization, and its lack of need of the annealing process [5].

The process has two steps, both of which are carried out in an autoclave at high temperatures and under high pressures. The first step is called the nucleation process. During this process, nuclear PZT crystals are deposited on the titanium base. The mechanism of crystal generation using the base material has an advantage with regard to the bonding of the film to the base material.

The original process did not contain titanium ions at the first step [5]. Thus, we proposed the improved processes [6,7] for electro-mechanical devices. The reaction conditions which were adopted for transducer fabrication are shown in Tables 1 and 2. About the process, there are two different ways for different applications. The process A indicated in Table 1 was applied to sensors [3]. This process is based on the single process [7] for the first step. Process B indicated in Table 2 was applied to micro-ultrasonic motors [8] and an ear phone [4]. Process $\mathrm{B}$ involves improved nucleation and crystal growth processes.

Table 1

Constituents of hydrothermal process A, called the improved single process

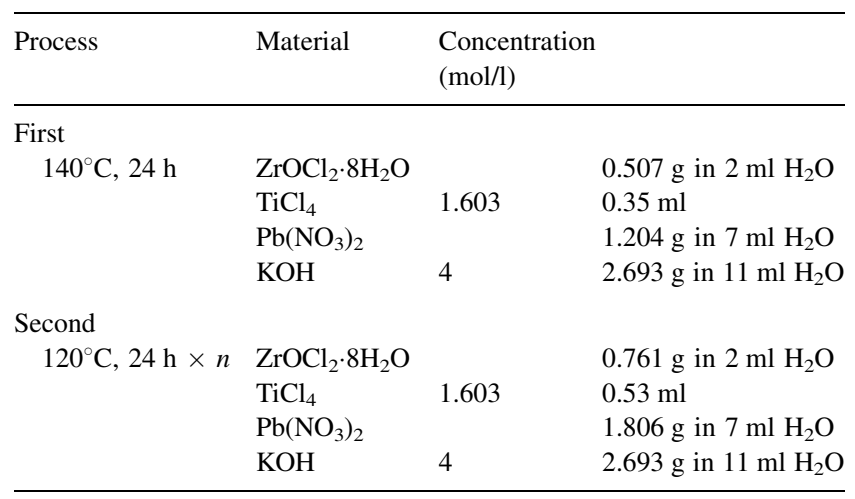

Table 2

Constituents of hydrothermal process $\mathrm{B}$, called the improved nucleation process

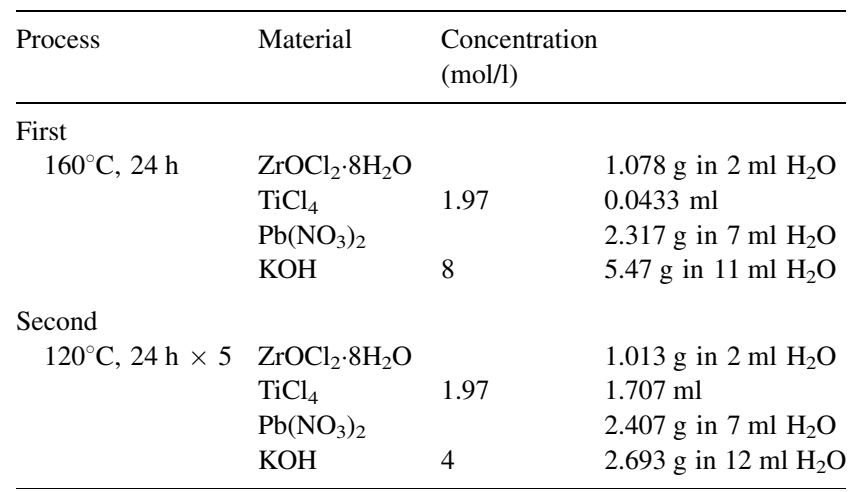

The second step process was carried out at a lower temperature of $120^{\circ} \mathrm{C}$, as indicated at Table 1 . During the second process, PZT crystals are expected to grow up. The thickness of the film increased by repeating the crystal growth process of process $\mathrm{A}$. In process $\mathrm{A}$, the thickness of the film is not saturated. After a single first step and four repetitions of the second step, the thickness reached a depth of $7 \mu \mathrm{m}$. However, in process $\mathrm{B}$, after the first step of nucleation process and four times second step of growth process, the thickness saturated in $12 \mu \mathrm{m}$.

The deposited film is crystallized as shown in Fig. 2, a scanning electron microscopy (SEM) photograph. This film was deposited using process $\mathrm{B}$. The polycrystal structure is a distinctive feature of the material of this process. The piezoelectric factor $d_{31}$ of deposited film was measured using the bimorph element $[6,9]$. Deformation was estimated based on

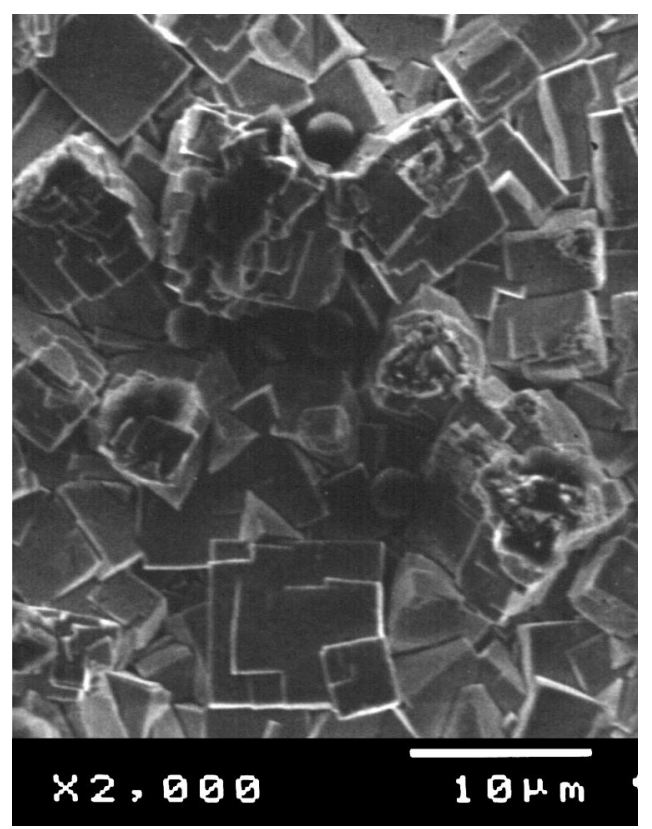

Fig. 2. SEM photograph of the hydrothermal PZT film surface. 
the measurement of the vibration amplitude at low frequency drive. The piezoelectric factor $d_{31}$ was estimated to be -34 or $-25 \mathrm{pC} / \mathrm{N}$ as based on the measurements. The bulk material factor is three times higher than these values [10].

\section{Sample transducers}

The sample transducers were rectangular in shape. These were longitudinal vibration mode transducers which were $7.9 \mathrm{~mm}$ long and $1 \mathrm{~mm}$ wide. The schematic shape of the longitudinal transducer is shown in Fig. 3. The vibration mode was half the wavelength of the longitudinal vibration. The element was supported at the center nodal point of the axial vibration component. The PZT film was deposited to a thickness of $7 \mu \mathrm{m}$ using the first and second step of process A, and to a thickness of $12 \mu \mathrm{m}$ using a single first step and four repetitions of the second step in process $\mathrm{B}$, on both sides of $100 \mu \mathrm{m}$ titanium base.

The photograph of the longitudinal transducer is shown in Fig. 4. The electrical supply was fed from the gold electrode deposited on the quarter wavelength part of one side and the titanium base.

The measurements of the vibration velocity were carried out using a laser Doppler vibrometer.

\section{Comparison of process types}

The maximum vibration velocity depends on material strength and is independent of frequency. We obtained a maximum vibration velocity of $0.53 \mathrm{~m} / \mathrm{s}$ by using a longitudinal vibrator for the PZT films used in the sensor application, and $0.9 \mathrm{~m} / \mathrm{s}$ by using a longitudinal vibrator for the PZT films used in the actuator application, as indicated in Fig. 5. The vibration velocity was not saturated, but the devices broke down due to the high electric field.

The maximum vibration level of the bulk PZT elements was $0.3 \mathrm{~m} / \mathrm{s}$ [11]. It has been recently reported that improved PZT with a dopant of $\mathrm{Fe}$ ions expresses better performance, but the maximum vibration velocity is around $0.6 \mathrm{~m} / \mathrm{s}$ under the condition wherein the $Q$-factor does not drop. In case of
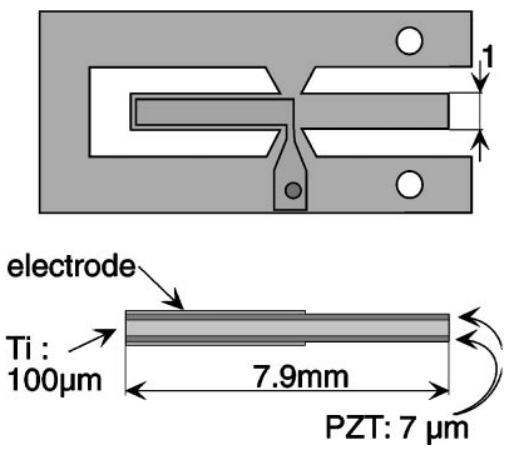

Fig. 3. Schematic view of the rectangular PZT transducer for longitudinal vibration; half wavelength.

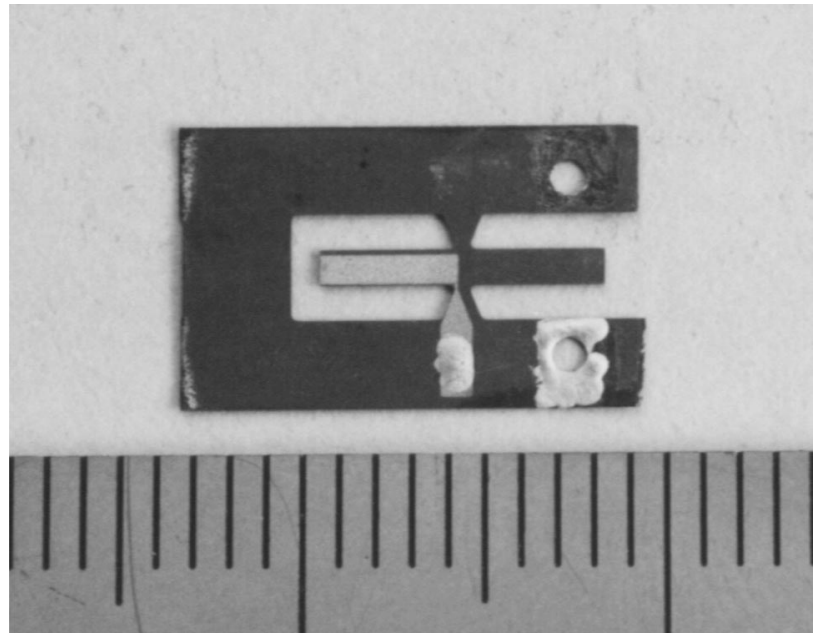

Fig. 4. Photograph of the rectangular transducer.

single crystal material such as $\mathrm{LiNbO}_{3}$, this limit is $3 \mathrm{~m} / \mathrm{s}$ [11]. Hydrothermal PZT material has characteristics midway between those of ceramic and single crystals.

\section{Influence of film thickness}

In process $\mathrm{A}$, film thickness increases as the growth process is repeated. The thickness increased up to 7,17 , 30 , and $38 \mu \mathrm{m}$ for each side of the titanium base after 2,5 , 10 , and 15 repetitions of the reaction process, i.e. the nucleation process and $1,4,9$, and 14 repetitions of the growth processes. This demonstrated that thicker PZT films can be created with higher repetitions of growth process A.

The SEM photographs of the film surface are shown in Fig. 6. These photos show the surface of the film after 2, 5, 10 , and 15 repetitions of the processes. From 2 to 5 repetitions, the crystals grew; however, after 10 and 15 repetitions,

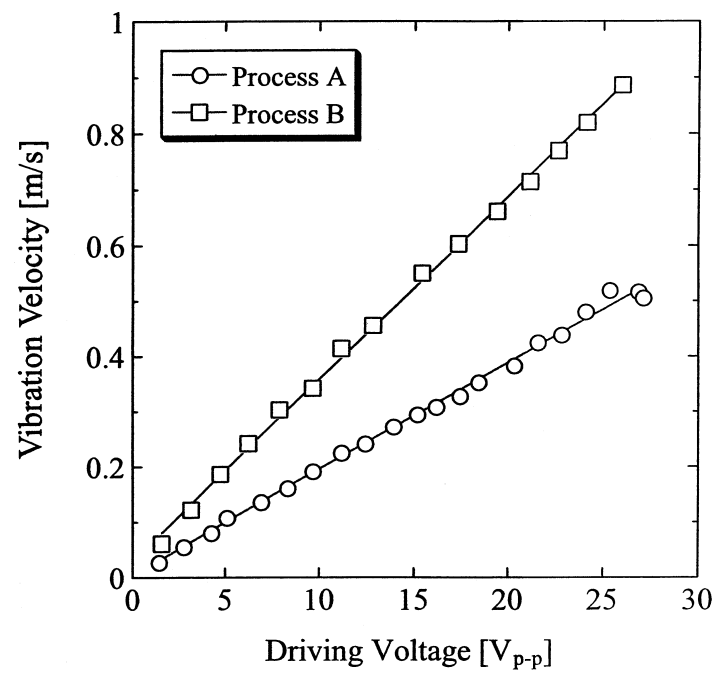

Fig. 5. Vibration velocities of the transducers deposited by processes A and $\mathrm{B}$. 

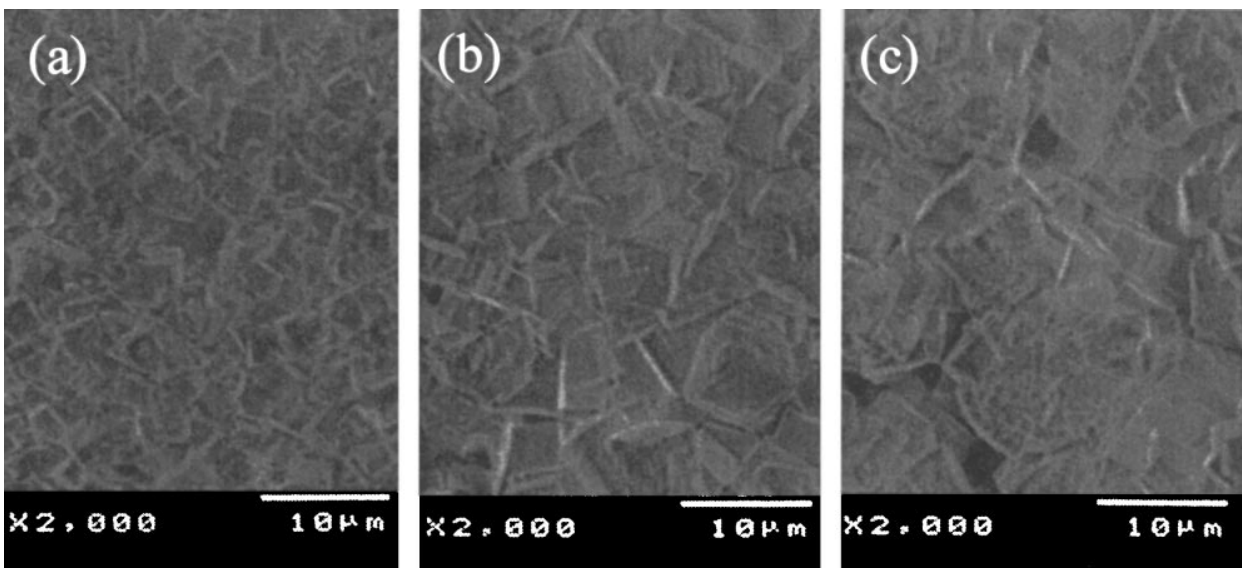

Fig. 6. SEM photographs of the surface of PZT films deposited using: (a) 2 repetitions of the deposition process; (b) 5 repetitions of the process, and (c) 15 repetitions of the process.

new crystals were obtained on the surface. The surface color of the film differs in films grown using 2 and 5 repetitions and in those using 10 and 15 repetitions. The 2 and 5 repetition films were black, while the 10 and 15 repetition films were light grey.

The vibration velocities of transducers using 2,5 , and 15 repetitions of the deposition process were measured. The results are shown in Fig. 7. The breakdown voltage increased as the growth process was repeated. In addition, the vibration velocity at the breakdown point increased from 0.53 to $0.90 \mathrm{~m} / \mathrm{s}$, and up to $2.24 \mathrm{~m} / \mathrm{s}$. The breakdown voltage was 26,58 , and $148 \mathrm{~V}_{\mathrm{p}-\mathrm{p}}$, respectively. The breakdown electric field was $1.3,1.7$, and $1.9 \mathrm{MV} / \mathrm{m}$, respectively.

There is not a large difference between the inclinations of plots. This result indicates that the piezoelectric constants are not strongly affected by the thickness of the film under this condition.

Fig. 8 shows the measured vibration velocity of a transducer using a film grown using 15 repetitions of the deposition process. Although, a first breakdown voltage was

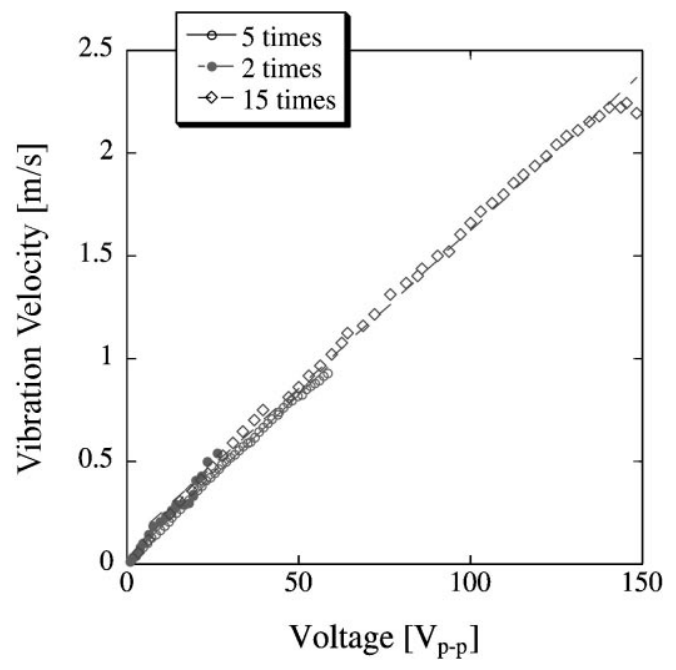

Fig. 7. Vibration velocities of the transducers using films created by the 2, 5 , and 15 repetitions processes.
$148 \mathrm{~V}_{\mathrm{p}-\mathrm{p}}$, a second breakdown occurred at a voltage of $188 \mathrm{~V}_{\mathrm{p}-\mathrm{p}}$. The reason for this cannot be explained precisely. However, it can be considered that the film had layered structure. If two layers, which have different properties, had been formed, there will be two different breakdown voltages. The deposition method consists of two processes which have two different conditions. Thus, it can be considered that there were two different film layers.

\section{Frequency response}

Under the condition of a constant driving voltage, the vibration velocity was measured under changing driving frequencies. In the case of bulk materials, it is well-known that the response curves are distorted and jumping phenomenon are observed $[12,13]$. In the case of hydrothermal PZT film devices, however, the response curves were smooth and continuous as shown in Figs. 9-11.

$Q$-factor of the element was about 400 up to a high intensity vibration level of $2.24 \mathrm{~m} / \mathrm{s}$ as shown in Figs. 911. This transducer was fabricated using process A to

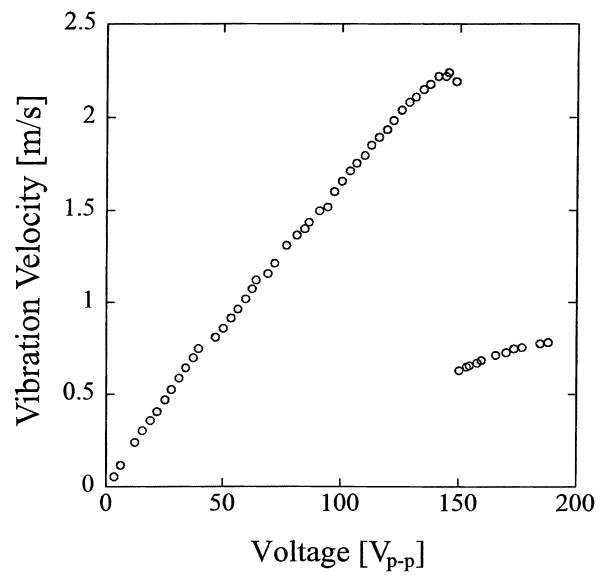

Fig. 8. Vibration velocity of the transducers using films created by the 15 repetitions process; $38 \mu \mathrm{m}$ thickness. 


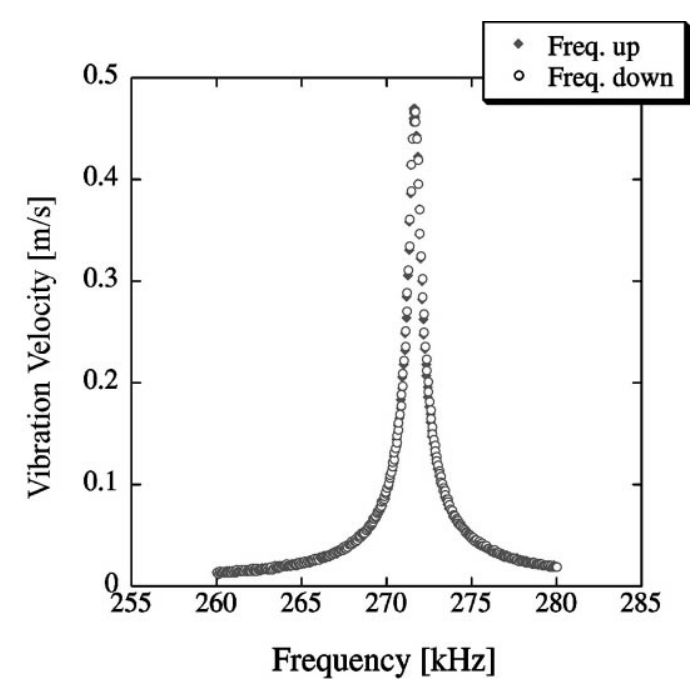

Fig. 9. Frequency response of the rectangular transducer at a low driving voltage of $25 \mathrm{~V}_{\mathrm{p}-\mathrm{p}}$.

deposit the PZT film. In these experiments, the frequency was changed upward and downward as indicated in the figures. In the case of bulk materials, it is well-known that the response curves at upward and downward frequencies have different traces. However, in the case of hydrothermal PZT, as shown in Figs. 9-11, the difference of the trace was small.

The resonance frequency depended on the strength of the driving electric field. At a lower driving voltage of $25 \mathrm{~V}_{\mathrm{p}-\mathrm{p}}$, as shown in Fig. 9, the resonance was higher than $271.5 \mathrm{kHz}$. However, it was $271.1 \mathrm{kHz}$ at the medium $63 \mathrm{~V}_{\mathrm{p}-\mathrm{p}}$, and was below $270.1 \mathrm{kHz}$ at $97 \mathrm{~V}_{\mathrm{p}-\mathrm{p}}$, as can be seen from Figs. 10 and 11. It seems that the piezoelectric factor and/or the elastic modulus changed with the driving voltage.

Regardless of the resonance frequency shift, the $Q$-factor of the transducer was constant against the driving voltage, and the response curves were very smooth and continuous.

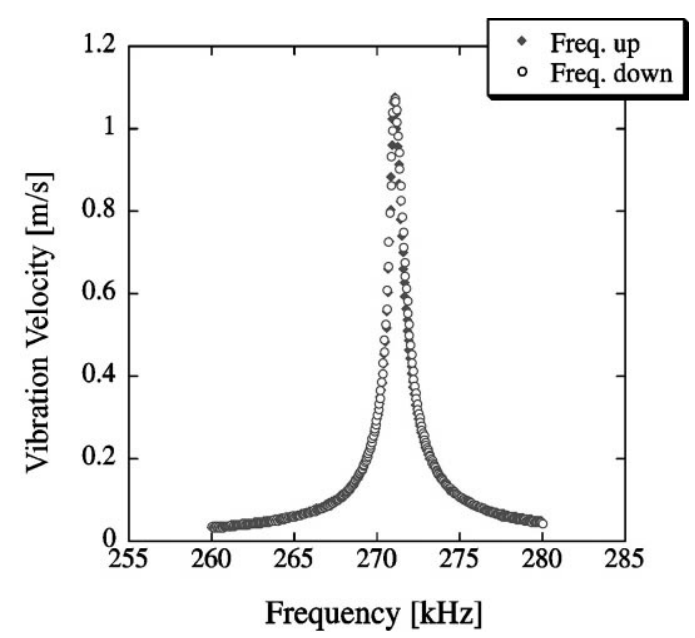

Fig. 10. Frequency response of the rectangular transducer at a middle driving voltage of $63 \mathrm{~V}_{\mathrm{p}-\mathrm{p}}$.

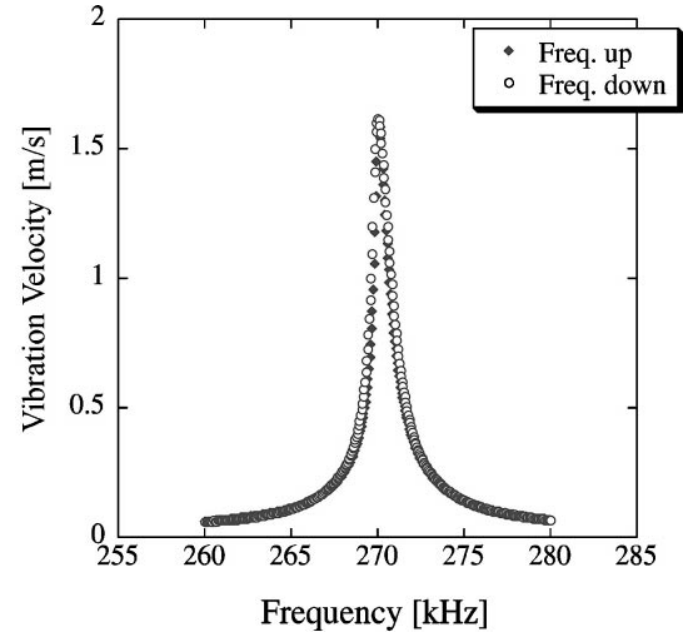

Fig. 11. Frequency response of the rectangular transducer at a high driving voltage of $97 \mathrm{~V}_{\mathrm{p}-\mathrm{p} \text {. }}$

The element seems to be applicable to sensor devices, and at the least it is superior to bulk PZT transducers which express strong nonlinearity.

\section{Piezoelectric constant}

The piezoelectric constant of the film can be estimated based on the vibration amplitude at the resonance frequency and $Q$-factor calculated by the resonance $Q$-curve. This is because the vibration amplitude at the resonance frequency equals the value of $Q$-factor times as large as the vibration amplitude when dc voltage is added.

When $t_{1}, t_{2}, Y_{\mathrm{s}}, c_{11}^{\mathrm{E}}, E_{3}$, and $S_{2}$ indicates the thickness of the titanium substrate and PZT film, the Yang's modulus of titanium substrate, the elastic stiffness of PZT film, the electric field, and the strain, respectively, the piezoelectric constant $e_{31}$ can be described as

$e_{31}=\frac{t_{1} Y_{\mathrm{s}}+2 t_{2} c_{11}^{\mathrm{E}}}{2 E_{3}} S_{2}$

This Eq. (1) can be derived from the motional equation and the piezoelectric equation

$T_{2}=c_{11}^{\mathrm{E}} S_{2}-e_{31} E_{3}$

where $T_{2}$ indicates stress.

From the calculation based on the experimental data, the piezoelectric coefficient $e_{31}$ of the longitudinal transducer was estimated to be $-0.34 \mathrm{C} / \mathrm{m}^{2}$ in process $\mathrm{A}$ and $-0.23 \mathrm{C} /$ $\mathrm{m}^{2}$ in process $\mathrm{B}$. These values are one-tenth of the constant of bulk materials.

Maximum stress can also be determined using the experimental data. When the film deposited using 15 repetitions was driven at $145 \mathrm{~V}_{\mathrm{p}-\mathrm{p}}$, the vibration amplitude was $1.34 \mu \mathrm{m}_{\mathrm{o}-\mathrm{p}}$. From Eq. (2), the maximum stress of this film was $14.8 \mathrm{MPa}$. 


\section{Conclusion}

The dynamic performance of hydrothermally-deposited PZT film transducers was measured. Two different deposition processes were tested to fabricate the longitudinal vibration mode transducers.

Linearity of the frequency response was excellent without the occurrence of the jumping effect and a decrease of $Q$ factors. The maximum vibration velocity was superior at $2.24 \mathrm{~m} / \mathrm{s}$ in longitudinal vibrator when the thickness of the film was $38 \mu \mathrm{m}$ and the breakdown voltage was $145 \mathrm{~V}_{\mathrm{p}-\mathrm{p}}$.

It is concluded that hydrothermal PZT has a high degree of linearity and high intensity output. These merits are effective for MEMS transducers. They seem to be based on the polycrystal structure of the material.

\section{Acknowledgements}

This work was supported by a grant-in-aid for general scientific research from the Ministry of Education, Science, Sports and Culture of Japan, and by the Proposal-Based New Industry Creative Type Technology R\&D Promotion Program from the New Energy and Industrial Technology Development Organization (NEDO) of Japan, and by a grant-in-aid for Research Fellowship for Young Scientists of the Japan Society for the Promotion of Science.

\section{References}

[1] T. Morita, M. Kurosawa, T. Higuchi, A cylindrical micro-ultrasonic motor fabricated by improved hydrothermal method, in: Proceedings of the International Conference on Solid-State Sensors and Actuators, Transducers '97, Chicago, June 1997, pp. 49-52.

[2] T. Kanda, T. Morita, M.K. Kurosawa, T. Higuchi, A rod-shaped vibro touch sensor using PZT thin film, IEEE Trans. Ultrasonic Ferroelectrics Freq. Cont. 46 (4) (1999) 875-882.

[3] T. Kanda, T. Morita, M.K. Kurosawa, T. Higuchi, A flat type touch probe sensor using PZT thin film vibrator, in: Proceedings of the Transducers '99, Sendai, June 1999, pp. 1508-1511.

[4] H. Yasui, M. Kurosawa, T. Morita, T. Kanda, T. Higuchi, Piezoelectric transducer for hearing aid using PZT thin film, in: Proceedings of the 16th International Congress on Acoustics, Seattle, 1998, pp. 2201-2202.

[5] K. Shimomura, T. Tsurumi, Y. Ohba, M. Daimon, Preparation of lead zirconate titanate thin film by hydrothermal method, Jpn. J. Appl. Phys. 30 (9B) (1991) 2174-2177.

[6] T. Morita, M.K. Kurosawa, T. Higuchi, A cylindrical microultrasonic motor using PZT thin film deposited by single process hydrothermal method, IEEE Trans. Ultrasonic Ferroelectrics Freq. Cont. 45 (5) (1998) 1178-1187.

[7] T. Morita, T. Kanda, M. Kurosawa, T. Higuchi, Single process to deposit lead zirconate titanate (PZT) thin film by hydrothermal method, Jpn. J. Appl. Phys. 36 (5B) (1997) 2998-2999.

[8] T. Morita, M.K. Kurosawa, T. Higuchi, A cylindrical shaped microultrasonic motor utilizing PZT thin film, in: Proceedings of the Transducers '99, Sendai, June 1999, pp. 1744-1747.
[9] Y. Ohba, M. Miyauchi, T. Tsurumi, M. Daimon, Analysis of bending displacement of lead zirconate titanate thin film synthesized by hydrothermal method, Jpn. J. Appl. Phys. 32 (9B) (1993) 40954098.

[10] B. Jaffe, W.R. Cook, H. Jaffe, Piezoelectric Ceramics, Academic Press, London, 1971.

[11] S. Hirose, K. Nakamura, et al., in: Proceedings of the Annual Meeting of Acoustical Society of Japan, 1991, pp. 845-846 (in Japanese).

[12] T. Wada, A study on jumping and dropping phenomena and their hysteresis of ceramic resonator, Trans. IIEJ 119 (3) (1999) 246-252 (in Japanese).

[13] J. Nosek, Drive level dependence of the resonant frequency in BAW quartz resonators and modeling, IEEE Trans. Ultrasonic Ferroelectrics Freq. Cont. 46 (4) (1999) 823-829.

\section{Biographies}

Takefumi Kanda was born in Fukuoka, Japan, on 18 June 1972. He received the $\mathrm{BE}$ and the $\mathrm{ME}$ degrees in precision machinery engineering from The University of Tokyo, Japan in 1997 and 1999, respectively. He is currently a doctoral student of the Graduate School of Engineering, The University of Tokyo. His research interests are micro-sensor and PZT thin film. He is a member of the Japan Society for Precision Engineering and the Institute of Electrical Engineers of Japan.

Minoru Kuribayashi Kurosawa (formerly Kuribayashi) was born in Nagano, Japan, on 24 April 1959. He received the BE degree in electrical and electronic engineering, and the $\mathrm{ME}$ and $\mathrm{PhD}$ degrees from Tokyo Institute of Technology, Tokyo, in 1982, 1984, and 1990, respectively. He was a Research Associate at the Precision and Intelligence Laboratory, Tokyo Institute of Technology, Yokohama, Japan, from 1984, and an Associate Professor at the Graduate School of Engineering, The University of Tokyo, Tokyo, Japan, from 1992. Since 1999, he has been an Associate Professor at the Interdisciplinary Graduate School of Science and Engineering, Tokyo Institute of Technology, Yokohama, Japan. His current research interests include ultrasonic motor, micro-actuator, PZT thin film, SAW sensor and actuator, and single bit digital signal processing and its application to control systems. Dr. Kurosawa is a member of the Institute of Electronics Information and Communication Engineers, the Acoustical Society of Japan, IEEE, the Institute of Electrical Engineers of Japan and the Japan Society for Precision Engineering.

Hidehiko Yasui was born in Kanagawa, Japan, on 6 May 1975. He received the BE and the ME degrees in precision machinery engineering from The University of Tokyo, Japan in 1998 and 2000, respectively. He is currently a doctoral student of the Graduate School of Engineering, The University of Tokyo. His research interests are electrostatic actuator, piezoelectric actuator and PZT thin film. He is a member of the Japan Society for Precision Engineering.

Toshiro Higuchi was born in Ehime, Japan on 26 February 1950. He received the $\mathrm{BE}, \mathrm{MS}$, and $\mathrm{PhD}$ degrees in precision engineering from The University of Tokyo, Japan, in 1972, 1974, and 1977, respectively. He was a Lecturer at the Institute of Industrial Science, The University of Tokyo in 1977, and an Associate Professor in the same institute from 1978. Since 1991, he has been a Professor at the Graduate School of Engineering, The University of Tokyo. His present interests include mechatronics, magnetic bearing, stepping motors, electrostatic actuator, robotics, and manufacturing. Dr. Higuchi is a member of the Japan Society for Precision Engineering, the Japan Society of Mechanical Engineers, and the Society of Instrument and Control Engineers. 